\title{
Acute Poisonings from Synthetic Cannabinoids - 50 U.S. Toxicology Investigators Consortium Registry Sites, 2010-2015
}

\author{
Anne M. Riederer, $\mathrm{ScD}^{1}$; Sharan L. Campleman, $\mathrm{PhD}^{1}$; Robert G. Carlson, PhD²; Edward W. Boyer, MD, PhD³; Alex F. Manini, MD ${ }^{4}$; \\ Paul M. Wax, MD ${ }^{1,5}$; Jeffrey A. Brent, MD, PhD ${ }^{1,6}$; Toxicology Investigators Consortium (ToxIC)
}

Recent reports suggest that acute intoxications by synthetic cannabinoids are increasing in the United States (1,2). Synthetic cannabinoids, which were research compounds in the 1980s, are now produced overseas; the first shipment recognized to contain synthetic cannabinoids was seized at a U.S. border in 2008 (3). Fifteen synthetic cannabinoids are Schedule I controlled substances (3), but enforcement is hampered by the continual introduction of new chemical compounds $(1,3)$. Studies of synthetic cannabinoids indicate higher cannabinoid receptor binding affinities, effects two to 100 times more potent than $\Delta^{9}$-tetrahydrocannabinol (the principal psychoactive constituent of cannabis), noncannabinoid receptor binding, and genotoxicity $(4,5)$. Acute synthetic cannabinoid exposure reportedly causes a range of mild to severe neuropsychiatric, cardiovascular, renal, and other effects $(4,6,7)$; chronic use might lead to psychosis $(6,8)$. During 2010-2015, physicians in the Toxicology Investigators Consortium (ToxIC) treated 456 patients for synthetic cannabinoid intoxications; 277 of the 456 patients reported synthetic cannabinoids as the sole toxicologic agent. Among these 277 patients, the most common clinical signs of intoxication were neurologic (agitation, central nervous system depression/coma, and delirium/toxic psychosis). Relative to all cases logged by 50 different sites in the ToxIC Case Registry, there was a statistically significant association between reporting year and the annual proportion of synthetic cannabinoid cases. In 2015, reported cases of synthetic cannabinoid intoxication increased at several ToxIC sites, corroborating reported upward trends in the numbers of such cases $(1,2)$ and underscoring the need for prevention.

In 2010, the American College of Medical Toxicology established the ToxIC Case Registry as a toxicology surveillance and research tool. Participating sites agree to record basic data on patients evaluated at local hospitals and clinics in cases where consultation by a medical toxicologist is requested; reported cases therefore represent severe or potentially severe toxicities. As of November 2015, there were active sites in 41 U.S. cities, with a few cities, such as Boston and New York City, having multiple sites. The registry is overseen by the Western Institutional Review Board and site-specific institutional review boards.

Temporal trends in the ToxIC synthetic cannabinoid case entries were investigated. Mixed logistic regression was used to evaluate the association between year and annual percentage of synthetic cannabinoid cases (among total cases, any agent), by site. The lme4 package in R (R Foundation for Statistical Computing, Vienna, Austria) was used to fit the model, accounting for intrasite and intragroup (e.g., participants in ToxIC's designer drug subregistry) correlation. To evaluate model fit, a deviance test was conducted, comparing the full model to a reduced model without the year variable. Sensitivity analyses were also conducted by dropping one site at a time and refitting the model.

During January 1, 2010-November 30, 2015, a total of 42,138 cases of toxic exposure were logged by 101 participating hospitals and clinics (Figure 1). Among these, 456 cases (at 50 ToxIC sites) involved synthetic cannabinoids, either as the sole toxicologic agent $(\mathrm{n}=277)$ or as a component of a multiagent exposure $(\mathrm{n}=179)$. Although most sites reported $<20$ synthetic cannabinoid cases, large sites in Harrisburg, Pennsylvania, New York City, Phoenix, Arizona, and Rochester, New York each recorded $\geq 30$ synthetic cannabinoid intoxication cases. In contrast, during the same period, only 13 cases were logged by ToxIC involving nonsynthetic cannabinoids (i.e., cannabis) as the sole toxicological agent; among these, the majority $(n=11)$ were children (aged 2-6 years) or teenagers (age 13-18 years).

Among all 456 synthetic cannabinoid intoxication cases, $322(70.6 \%)$ occurred in persons aged $19-65$ years and $125(27.4 \%)$ occurred in persons aged $13-18$ years; 379 $(83.1 \%)$ patients were male. The most common street names of synthetic cannabinoids reported by patients or accompanying friends and family members were $\mathrm{K} 2$ and Spice. In 415 (91.0\%) cases, the patient had clinical signs or symptoms of intoxication; specific toxicologic treatments were administered to $267(58.6 \%)$ patients, whereas the rest received standard supportive care and monitoring before being discharged. No specific synthetic cannabinoid antidotes exist.

Among the 277 (61\%) patients who reported synthetic cannabinoids as the sole toxicologic exposure, the system most commonly affected was the central nervous system (Table), manifested by agitation, central nervous system depression/ coma, and delirium/toxic psychosis, with seizures and hallucinations reported less frequently. Information on death during hospitalization was available for 246 (54\%) patients. Among these, three $(1.2 \%)$ deaths were recorded. The first occurred in 
FIGURE 1. Toxicology Investigators Consortium (ToxIC)* registry cases caused by all agents and by synthetic cannabinoid, ${ }^{\dagger}$ by U.S. registry site location ${ }^{\S}$ - January 1, 2010-November 30, 2015

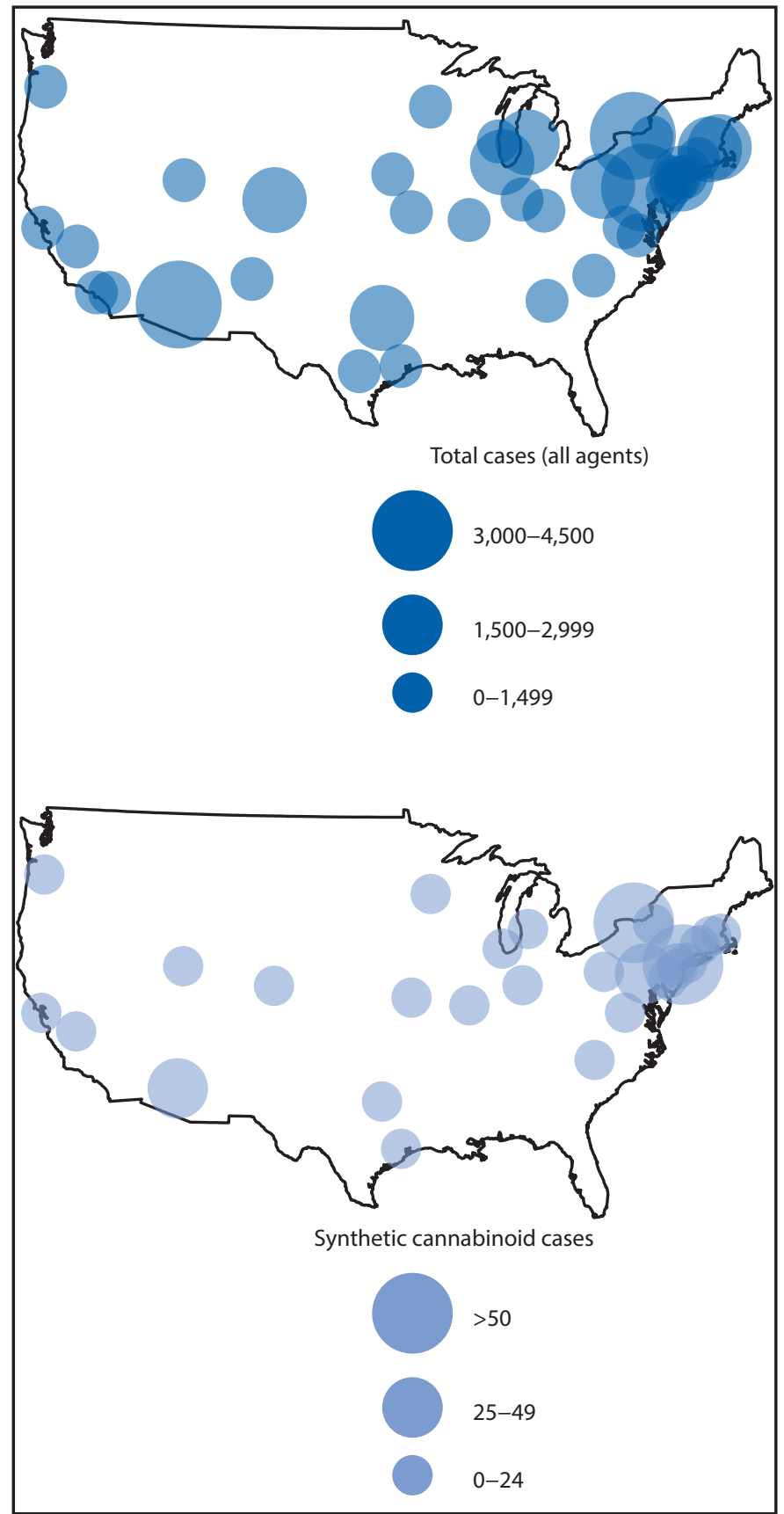

* ToxIC is a select, volunteer network and thus not geographically representative of the United States or the cities where participating sites are located; many sites joined ToxIC after its establishment in 2010 by the American College of Medical Toxicology.

${ }^{\dagger}$ As primary agent or part of multiagent exposure.

$\S$ As of November 2015, there were active ToxIC registry sites in 41 U.S. cities, with a few cities (e.g., Boston and New York City) having multiple sites.
TABLE. Percentage of patients $(n=277)$ reporting synthetic cannabinoids as the sole toxicologic agent* among 42,138 cases of toxic exposure reported at 101 participating hospitals and clinics, by clinical sign or symptom - Toxicology Investigators Consortium (ToxIC) registry, January 1, 2010-November 30, 2015

\begin{tabular}{llc}
\hline $\begin{array}{l}\text { Organ system/ } \\
\text { Syndrome }\end{array}$ & \multicolumn{1}{c}{$\begin{array}{c}\text { Clinical } \\
\text { sign/symptom }\end{array}$} & $\begin{array}{c}\text { Patients } \\
\text { reporting SC } \\
\text { Nervous }\end{array}$ \\
Cardiovascular & Agitation, coma, toxic psychosis, other & (\%) \\
Pulmonary & Bradycardia, tachycardia, other & 66.1 \\
& Respiratory depression & 17.0 \\
Renal/Muscle & Other & 5.4 \\
& Acute kidney injury & 2.2 \\
Other & Rhabdomyolysis & 4.0 \\
& Metabolic & 6.1 \\
& Gastrointestinal/Hepatic & 8.7 \\
Toxidrome & Significant leukocytosis & 1.4 \\
& Sedative-hypnotic & 2.9 \\
& Sympathomimetic syndrome & 6.9 \\
& Other & 5.4 \\
\hline
\end{tabular}

Abbreviation: SC = synthetic cannabinoid.

* A total of 456 reported cases (at 50 ToxIC sites) involved synthetic cannabinoids, either as the sole toxicologic agent $(n=277)$ or as a component of a multiagent exposure $(n=179)$.

† Percentages do not sum to $100 \%$ because some patients had more than one clinical sign.

a male aged 17 years, who suffered a cardiac arrest after reportedly taking a single "hit" of K2/Spice; the second occurred in an adult male with respiratory depression, agitation, and delirium/toxic psychosis after allegedly taking a synthetic cannabinoid and oxycodone; and the third occurred in an adult male with similar signs, who developed acute kidney injury after reportedly taking a synthetic cannabinoid, a synthetic cathinone (commonly known as bath salts), and the psychedelic drug lysergic acid diethylamide (LSD).

During 2010-2015, the annual percentage of synthetic cannabinoid cases among sites increased in all four U.S. Census regions; during 2014-2015, the annual percentage increased in all regions except the South (Figure 2). The largest overall increases during these periods took place in the Northeast, primarily driven by increases at the New York City sites. Less distinct but discernable increases occurred at sites in several other cities nationwide, and a decrease occurred at the Rochester, New York, site; heterogeneous patterns occurred elsewhere (not shown). In the mixed regression analysis, the deviance test indicated that including year in the model provided a significantly $(\mathrm{p}<0.05)$ better fit, evidence of a statistically significant temporal trend. In the sensitivity analyses, including the year variable improved model fit in a statistically significant manner, in each iteration (i.e., when the model was refit after dropping one site at a time). 
FIGURE 2. Percentage of reported ToxIC* registry cases ${ }^{\dagger}$ caused by synthetic cannabinoids, by U.S. Census region - 2010, 2014, and 2015

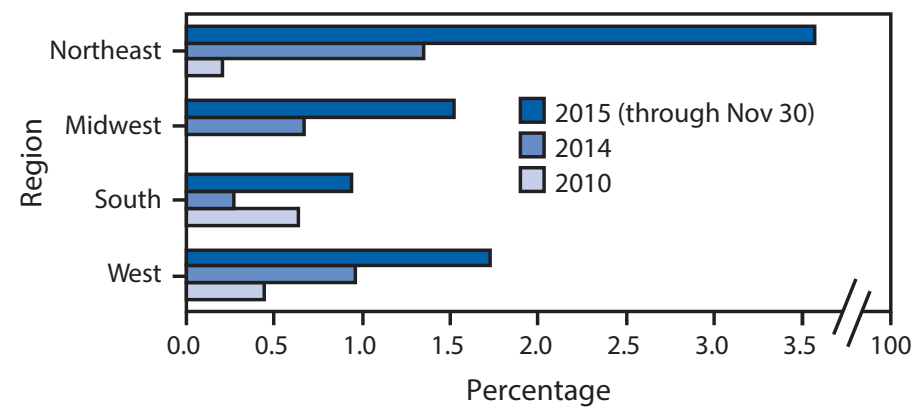

* ToxIC is a select, volunteer network and thus not geographically representative of the United States or the cities where participating sites are located; many sites joined ToxIC after its establishment in 2010 by the American College of Medical Toxicology.

† Includes only cases from sites that reported any synthetic cannabinoid cases.

\section{Discussion}

The ToxIC data complement data from health agencies, poison centers, and other sources to produce a more detailed picture of the acute public health impacts of synthetic cannabinoid use in the United States. Although some potential for report overlap exists, cases in the ToxIC Registry are not routinely reported to poison centers. The significant increase in synthetic cannabinoid poisonings identified through this consortium reflects recent trends, which include a Drug Enforcement Agency report of 22 synthetic cannabinoid clusters (including two deaths) and 25 additional episodes (including 18 deaths) in 25 states during August 2011-April 2015 (1), as well as a 330\% increase in synthetic cannabinoid-related calls to U.S. poison centers during the first 4 months of 2015 (2). The observed increases might result from increased synthetic cannabinoid use; the appearance of more toxic and potent synthetic cannabinoid compounds or multisynthetic cannabinoid formulations; increased recognition of synthetic cannabinoids as a cause of acute poisoning; increased familiarity among medical personnel with the clinical signs and symptoms of synthetic cannabinoids; or a combination of these factors $(6,7)$.

The findings in this report are subject to at least five limitations. First, although ToxIC is a unique tool, it is clinically based, not population-based, and thus is not geographically representative of the United States or the cities where participating sites are located. The consortium includes most U.S. medical toxicology clinical services, but large areas of the country that do not have direct access to medical toxicologists are underrepresented. Second, although the consortium strives to report all cases treated by medical toxicologists at participating sites, reporting might be affected by several factors, including clinical caseload, personnel changes, and referral patterns. Nonetheless, the consortium's use of normalized

\begin{abstract}
Summary
What is already known about this topic?

Acute intoxications by synthetic cannabinoids appear to be increasing in the United States. Synthetic cannabinoids are two to 100 times more potent than $\Delta^{9}$-tetrahydrocannabinol, the active ingredient in cannabis; acute exposure is associated with a range of mild to severe neuropsychiatric, cardiovascular, renal, and other effects.

What is added by this report?

During 2010-2015, among 456 cases of synthetic cannabinoid intoxication among patients treated by U.S. medical toxicologists, 277 (61\%) had reports of synthetic cannabinoids as the sole toxicologic agent. Three deaths were recorded, one with synthetic cannabinoids given as the sole agent and two with multiple agent exposures. Synthetic cannabinoid poisonings increased in all U.S. Census regions.

What are the implications for public health practice?

The increase in acute synthetic cannabinoid poisonings underscores the importance of targeted prevention interventions and the need for education about the potentially lifethreatening consequences of synthetic cannabinoid use.
\end{abstract}

statistics (the proportion of all consultations that were related to synthetic cannabinoids) and a mixed regression approach, which accounts for intrasite variability, improves confidence that the observed temporal increases are real. Third, synthetic cannabinoid case identification was based on patient history and clinical presentation; analytical confirmation is not available for most synthetic cannabinoid cases in the registry. The development of analytical tests that reliably detect synthetic cannabinoids and their metabolites in biologic samples is hindered by the production of new chemical compounds for which no analytical standards exist, difficulties in finding unique synthetic cannabinoid biomarkers, and other challenges $(2,9,10)$; thus, analytical tests are not routinely used by every ToxIC physician. Instead, these physicians rely on patient self-reports or reports of accompanying family members or friends. Because of this, reports of drugs taken might be inaccurate, leading to misattribution of certain clinical signs and symptoms to synthetic cannabinoids. Fourth, as is common in drug abuse/misuse cases (8), approximately half of the ToxIC synthetic cannabinoid cases involved multiagent exposures, including synthetic cannabinoids in combination with other illicit or prescription drugs or alcohol. Consequently, other agents, or the combination of psychoactive substances, might have been responsible for the effects observed. A small German study, with analytical confirmation of the synthetic cannabinoids and other drugs in patient samples, reported that clinical signs in patients with concurrent drug exposures were similar to those who were exposed only to synthetic cannabinoids (7). Finally, patients occasionally declined to divulge details 
of their exposure. For example, among 37,984 total cases recorded at ToxIC's U.S. sites during January 1, 2010-June 30, 2015 , a total of $3,153(8.3 \%)$ were missing agent information or recorded as unknown agent. Some of these cases possibly involved synthetic cannabinoids but were not recorded as such.

The increase in acute synthetic cannabinoid poisonings observed in ToxIC underscores the need for targeted prevention interventions. Educating the public on the potentially life-threatening consequences of synthetic cannabinoid use is important for countering the observed upward trend in synthetic cannabinoid poisonings.

\section{Acknowledgments}

Toxicology Investigators Consortium members and personnel.

${ }^{1}$ ToxIC, American College of Medical Toxicology, Phoenix, Arizona; ${ }^{2}$ Boonshoft School of Medicine, Wright State University, Dayton, Ohio; ${ }^{3}$ University of Massachusetts Medical School, Worcester, Massachusetts; ${ }^{4}$ Division of Medical Toxicology, The Icahn School of Medicine at Mt. Sinai, New York, New York; ${ }^{5}$ University of Texas Southwestern Medical Center, Dallas, Texas; ${ }^{6}$ Department of Medicine, University of Colorado School of Medicine, Colorado School of Public Health, Aurora, Colorado.

Corresponding author: Anne M. Riederer, anne.riederer@acmt.net, 844-226-8333, ext. 710 .

\section{References}

1. Trecki J, Gerona RR, Schwartz MD. Synthetic cannabinoid-related illnesses and deaths. N Engl J Med 2015;373:103-7. http://dx.doi. org/10.1056/NEJMp1505328
2. Law R, Schier J, Martin C, Chang A, Wolkin A. Increase in reported adverse health effects related to synthetic cannabinoid use-United States, January-May 2015. MMWR Morb Mortal Wkly Rep 2015;64:618-9.

3. US Drug Enforcement Administration Office of Diversion Control. Schedules of controlled substances: temporary placement of three synthetic cannabinoids into Schedule I. 21 C.F.R. Part 1308 (2015). http://www.deadiversion.usdoj.gov/fed_regs/rules/2015/fr0130.htm

4. Castaneto MS, Gorelick DA, Desrosiers NA, Hartman RL, Pirard S, Huestis MA. Synthetic cannabinoids: epidemiology, pharmacodynamics, and clinical implications. Drug Alcohol Depend 2014;144:12-41. http://dx.doi.org/10.1016/j.drugalcdep.2014.08.005

5. Koller VJ, Ferk F, Al-Serori H, et al. Genotoxic properties of representatives of alkylindazoles and aminoalkyl-indoles which are consumed as synthetic cannabinoids. Food Chem Toxicol 2015;80:1306. http://dx.doi.org/10.1016/j.fct.2015.03.004

6. van Amsterdam J, Brunt T, van den Brink W. The adverse health effects of synthetic cannabinoids with emphasis on psychosislike effects. J Psychopharmacol 2015;29:254-63. http://dx.doi. org/10.1177/0269881114565142

7. Hermanns-Clausen M, Kneisel S, Szabo B, Auwärter V. Acute toxicity due to the confirmed consumption of synthetic cannabinoids: clinical and laboratory findings. Addiction 2013;108:534-44. http://dx.doi. org/10.1111/j.1360-0443.2012.04078.x

8. Hurst D, Loeffler G, McLay R. Psychosis associated with synthetic cannabinoid agonists: a case series. Am J Psychiatry 2011;168:1119. http://dx.doi.org/10.1176/appi.ajp.2011.11010176

9. Castaneto MS, Wohlfarth A, Desrosiers NA, Hartman RL, Gorelick DA, Huestis MA. Synthetic cannabinoids pharmacokinetics and detection methods in biological matrices. Drug Metab Rev 2015;47:124-74. http://dx.doi.org/10.3109/03602532.2015.1029635

10. Znaleziona J, Ginterová P, Petr J, et al. Determination and identification of synthetic cannabinoids and their metabolites in different matrices by modern analytical techniques — a review. Anal Chim Acta 2015;874:1125. http://dx.doi.org/10.1016/j.aca.2014.12.055 